\title{
LES ACTEURS DE L'INTELLIGENCE ÉCONOMIQUE DANS LES ENTREPRISES : LE CAS DES ENTREPRISES EUROPÉENNES DE HAUTES TECHNOLOGIES
}

\author{
David SALVETAT* \& Zouhair LAARRAF**
}

\begin{abstract}
Résumé - Les acteurs de l'intelligence économique (IE) demeurent peu identifiés dans les organisations actuelles. Les études antérieures s'opposent quant à l'identification des personnes ayant en charge la réalisation du processus d'IE. Forte de ce constat, cette étude tend à cartographier les salariés qui sont directement ou indirectement participatifs à cette démarche de renseignement au sein des entreprises. En d'autres termes, qui pratique l'IE dans l'entreprise ? Pour répondre à cette question, une étude empirique a été menée sur un échantillon de 153 entreprises des industries de hautes technologies en Europe. Les résultats obtenus indiquent que le rôle du dirigeant est essentiel, mais que les cadres sont les individus qui participent très fortement au processus d'IE avec les experts de l'information. Nos conclusions montrent néanmoins que les salariés restent peu participatifs à cette démarche.
\end{abstract}

Mots clés: Intelligence économique; Acteurs; ressources humaines ; expertise informationnelle.

\section{Introduction}

Issu du monde militaire, le renseignement économique s'est transformé tant sur ses pratiques que sur son idéologie. L'intelligence économique (IE) est un ensemble d'actions coordonnées de recherche, de collecte, d'analyse et de diffusion de l'information (Martre, 1994, Chung et al., 2002). Certaines études considèrent que la veille stratégique et l'IE sont similaires. D'autres travaux indiquent néanmoins que les phases de recherche et de collecte constituent la veille. Cette dernière ainsi que les phases d'analyse et de diffusion constituent le processus complet d'IE (Lackman et al., 2000, Chung et al., 2002). Dans notre étude, nous

* Professeur - HDR Groupe Sup de Co La Rochelle - CEREGE 102, rue des Coureilles 17000 La Rochelle salvetatd@esc-larochelle.fr

** Professeur associé Groupe Sup de Co La Rochelle - CEREGE 102, rue des Coureilles 17000 La Rochelle laarrafz@esc-larochelle.fr 
distinguerons la veille de l'IE. D'abord étudié dans les grandes entreprises, le concept d'IE acquiert une dimension stratégique forte (Baumard, 2000) dans les PME. Phase appartenant au processus de décision, c'est un outil de management à part entière (Prescott, 1999) visant à améliorer la compétitivité de l'entreprise (Bournois et Romani, 2000). Passerelle interactive entre environnement et entreprise (Dou, 1995), ce processus s'appuie sur l'animation de réseaux internes et externes (Lönnqvist et Pirttimäki, 2006). L'IE n'est plus seulement un outil de gestion et d'analyse informationnelle, mais elle consiste aussi à entreprendre un certain nombre d'actions dans le but d'influencer son environnement (Porter, 1985, Prescott, 1995).

L'organisation des activités de renseignement conditionne la viabilité ou non de la démarche d'IE. Structurer et gérer les activités de surveillance peuvent se réaliser de diverses manières. Toutefois, une démarche d'IE est plus ou moins structurée dans une entreprise. Dans les écrits, cette structuration s'appréhende selon la centralisation/décentralisation, l'internalisation/externalisation et la formalisation/non formalisation du processus d'IE. Bien que ces trois couples d'opposition soient unanimement reconnus par les auteurs, leurs conclusions et leurs visions théoriques différent. Certains préconisent une internalisation, une centralisation et une formalisation du processus, alors que d'autres recommandent l'inverse. Ce premier constat constitue une limite dans l'attribution des tâches de chaque salarié dans l'effort de surveillance.

$\mathrm{Au}$ cœur des enjeux de l'entreprise, l'IE concerne de prime abord l'ensemble des salariés dans l'entreprise. L'IE est une démarche collective et participative, quel que soit le niveau hiérarchique des salariés, qui ne trouve son efficacité que dans la contribution de tous. Le rapport qu'ont les salariés avec l'information est une clé de la performance de la firme et de l'avantage concurrentiel. Le dirigeant d'entreprise doit alors insuffler la volonté de pratiquer l'IE. Il sensibilise les salariés, les motive et légitime le processus d'IE.

L'établissement d'une culture du renseignement devient inévitable dans l'entreprise afin de construire et de protéger son patrimoine informationnel. Au regard des travaux académiques, l'ensemble des auteurs préconise la participation des salariés à la démarche d'IE. Néanmoins, les résultats de ces mêmes travaux corroborent peu cette recommandation. De plus, nombre d'études antérieures s'opposent dans leurs observations et conclusions. Certains auteurs indiquent qu'il est inutile d'impliquer l'ensemble des salariés au risque de diminuer la qualité de la démarche au détriment d'un processus efficace mené par des experts de l'IE. Par conséquent, les travaux répondent peu à la question : qui pratique l'IE dans l'entreprise?

Dans cette étude, nous reprenons cette problématique afin de tenter de cartographier les acteurs des activités de surveillance dans les entreprises. Une étude empirique est menée sur un échantillon de 153 entreprises des industries de hautes technologies en Europe. Les résultats obtenus indiquent que le rôle du dirigeant est essentiel, mais que les cadres sont les individus qui participent très fortement au processus d'IE avec les experts de l'information. Nos conclusions montrent néanmoins que les salariés restent peu participatifs à cette démarche. 


\section{Fondements théoriques}

\subsection{La place et le rôle de l'information dans le management des ressources humaines}

L'information occupe une place essentielle au sein du management des ressources humaines. L'approche par la théorie des ressources offre, en ce sens, un éclairage intéressant. Ressources informationnelles et ressources humaines constituent les pivots d'une démarche d'IE intégrée dans l'entreprise. Une ressource est qualifiée de stratégique si elle est rare, inimitable, non substituable, non transférable et valorisable (modèle VRIO). Elle doit permettre un accès à un grand nombre de marchés et contribuer à la valeur du produit final (Hamel et Prahalad, 1995). Elle doit être difficilement imitable et rare, ce qui suppose l'existence d'une ambiguïté causale (Reed et DeFillippi, 1990) qui empêche les imitateurs potentiels de savoir quoi imiter et comment le faire. Elle maintient une difficulté pour relier une cause et une conséquence, et s'apprécie en fonction notamment de son aspect tacite et de sa complexité. Elle doit être difficilement substituable et transférable (Collis et Montgomery, 1995), afin de se situer hors de la portée d'un éventuel processus de création destructrice selon une approche Schumpeterienne. La conception de la place de l'information au sein des ressources humaines s'inscrit pleinement dans l'héritage de Penrose (1956).

Concernant l'approche classique des ressources, ces dernières sont les plus pertinentes lorsqu'elles sont les plus cohérentes à un environnement spécifique. Ce raisonnement par écarts de ressources montre que la nature et la qualité des ressources détenues permettent l'obtention d'une rente (Peteraf, 2006), pérennisant ainsi un avantage concurrentiel. Les salariés doivent être conscients qu'une information est une ressource stratégique dès lors qu'elle répond aux critères du modèle VRIO. La sensibilisation et la formation des différents acteurs de l'entreprise deviennent essentielles lorsqu'ils participent directement ou indirectement à une démarche d'IE ayant comme socle l'information.

Pour le modèle d'analyse stratégique (ISPC : intention stratégique et pôle de compétence), la captation de ressources manquantes et la valorisation des ressources existantes constituent deux axes clés pour une firme (Desreumaux, 1993). En ce sens, l'intégration d'une démarche d'IE a pour vocation de se prémunir des fuites informationnelles et de capitaliser les informations internes et externes créatrices de savoirs et de valeur. L'information devient un patrimoine de l'entreprise qu'il convient de faire fructifier, de pérenniser, de transmettre et de protéger. Chaque salarié est garant de cette démarche.

S'agissant de l'approche par les capacités dynamiques des entités et l'apprentissage organisationnel, (Teece et al., 2004), les ressources et compétences internes s'intègrent, se construisent et se reconfigurent selon l'évolution de l'environnement de l'entreprise. Les capacités dynamiques se forment à travers des mécanismes de l'apprentissage (Eisenhardt et Martin, 2000). La participation des salariés au management des informations permet une remise en cause constante face aux transformations externes. Le management des informations et l'IE rendent alors possible un réel apprentissage généré par les actions des acteurs composant l'entreprise.

Comme le soulignent Becker et Huselid (2006), la GRH est un gisement de la performance pour les organisations. Beaupré et Cloutier (2007) indiquent que les pratiques RH mobilisatrices auraient l'aptitude d'intensifier la performance économique et sociale de l'entreprise, en stimulant l'engagement et en développant les compétences des salariés. Pour 
Huselid (2011), ces pratiques agiraient à trois niveaux : sur l'engagement (la volonté d'agir), sur les compétences requises (le pouvoir agir) et sur les possibilités de mettre en pratique ces compétences (l'opportunité d'agir). Les pratiques mobilisatrices sont : l'investissement en formation; la mise en œuvre d'une organisation de travail plus organique ; l'encouragement de la participation ; le partage de l'information et l'instauration d'une rémunération liée à la performance.

Chaque salarié devient acteur et client au management de l'information. L'information devient alors une ressource intégrée à la gestion des ressources humaines. Cette dernière s'insère dans de nouvelles pratiques de veille, de maîtrise de l'information, etc. Par conséquent, la structuration de l'IE devient un axe essentiel dans une politique RH.

\subsection{Structuration de l'IE et organisation}

Le processus d'IE accepte principalement trois modes d'intégration dans l'entreprise, qui sont autant de débats dans les travaux académiques : la centralisation/décentralisation, l'internalisation/externalisation et la formalisation/non formalisation de la démarche d'IE. Toutefois, ces modes dépendent fortement de facteurs contingents internes (taille, ressources, etc.) et externes (secteur, lois, etc.).

Les pratiques d'IE centralisée sont majoritairement observées dans les entreprises (Bournois et Romani, 2000, Levet, 2002, Salles, 2003). Néanmoins, la veille reste parfois considérée comme une pratique évidente, menée de manière inconsciente par chaque salarié. Pour Levet (2002), 39 \% des firmes se renseignent sans avoir de cellule de veille. La fonction d'IE est souvent centralisée au niveau du dirigeant de PME, alors qu'elle est plutôt décentralisée auprès d'experts de l'information dans les grandes structures (Pearce, 1982). La centralisation de l'IE permet une vision globale, une satisfaction rapide des besoins en information, une facilitation des analyses, des duplications réduites, des ressources internes non gaspillées et moins limitées, un personnel motivé, disponible et formé, etc. La centralisation de l'IE est donc à privilégier (Porter, 1985) afin d'acquérir une légitimité de la démarche d'IE auprès des salariés. Pour Lackman et al. (2000), l'IE est centralisée dans $46 \%$ des entreprises, surtout au niveau de la Direction Générale à $55 \%$ (Bournois et Romani, 2000). Stubbart (1982) préconise plutôt une IE décentralisée au sein de chaque fonction. Une décentralisation de l'IE permet en effet une expertise multi-acteurs et multi domaines, des informations recueillies mieux ciblées aux besoins, et une surveillance plus opérationnelle et intégrée au processus de décision.

L'IE traite de l'information qui peut se révéler stratégique pour la firme. Pour cette raison, l'IE reste essentiellement internalisée. Les informations sont dirigées vers des experts qui auront à les traiter et à juger de leur pertinence. Les auteurs restent cependant partagés sur cette nécessité d'internalisation. Certaines activités de renseignement sont externalisées auprès de prestataires spécialisés. La veille est surtout concernée, car elle se limite à une simple collecte d'informations. Le recours à des prestataires constitue un complément à une démarche d'IE existante interne.

Fahey et King (1977) recommande une entité d'IE interne et autonome. Lackman et al. (2000) vont plus loin en préconisant la constitution de trois groupes d'individus : un premier dédié à la collecte et au filtrage des informations, un deuxième dédié à la construction et à l'entretien des données utiles, et un dernier dédié à l'analyse des informations et à la 
planification stratégique. Pour Jain (1984) et Prescott, (1999), environ $30 \%$ des entreprises étudiées ont une cellule interne. Ce taux dépasse les $52 \%$ pour d'autres études.

Le processus d'IE utilise aussi bien des informations formelles (écrites) qu'informelles (orales). Dans les entreprises, le degré de formalisation reste corrélé avec le niveau d'avancement de la surveillance (Porter, 1985). Phanuel (2002) mesure les procédures formalisées au sein de PME/ PMI, selon les phases du processus d'IE : 17,5\% pour la collecte des informations ; $22,5 \%$ pour leur analyse et $50,5 \%$ pour leur diffusion. Peters et Waterman (1984) montrent que $72 \%$ des managers souhaitent une formalisation des informations. Pour Bournois et Romani (2000), seulement 12,4 \% des firmes ont une démarche formalisée, tandis que pour Diffenbach (1983), $73 \%$ des entreprises ont formalisé leur activité de surveillance. Pour Preble et al. (1988), ce taux est de $51 \%$; et il est largement supérieur à $50 \%$ pour Klein et Linneman (1984). La formalisation de l'IE permet de meilleure qualité, quantité, ciblage, rentabilité et fiabilité des informations (Gilad et Gilad, 1985). La non formalisation permet néanmoins de réduire les coûts d'organisation, de fonctionnement et de formation des individus. De plus, une formalisation forte peut être perçue comme un outil de contrôle par les salariés, et un manque de créativité de part sa standardisation, limitant ainsi l'avantage concurrentiel.

$\mathrm{Au}$ regard de ce qui précède, la pratique de l'IE est plurielle. Terry (1977) indique qu'un compromis doit être trouvé entre centralisation/décentralisation, internalisation/externalisation et formalisation/non formalisation. Les entreprises peuvent disposer à la fois d'une entité spécialisée au niveau du groupe, et de structures décentralisées de surveillance au niveau des divisions ou des fonctions. Les cellules supports seraient ainsi rattachées à la cellule centrale (Gilad et Gilad, 1985).

\subsection{Les Ressources Humaines : principaux acteurs de l'IE}

Le dirigeant d'entreprise insuffle la volonté de pratiquer l'IE. Il assure, auprès de ses salariés, une certaine transmission des savoirs et savoir-faire. Il inculque à l'ensemble des salariés la culture du renseignement. Son rôle est de proposer des formations, des incitations financières, sociales, etc. L'IE est en effet une démarche collective. Il donne les moyens et encourage tous les salariés, chacun dans sa spécialité, à effectuer une veille. L'IE est alors une démarche participative, à tous les niveaux hiérarchiques et quelle que soit la fonction du salarié (Rouach, 2008).

L'IE ne peut se pratiquer sans l'implication des collaborateurs qui composent l'entreprise (Achard, 2005). Les politiques managériales sensibilisent et rendent légitimes l'IE auprès des ressources humaines. L'IE est un service délivré aux clients internes. Elle nécessite une réelle pédagogie afin de susciter l'intérêt de la compréhension des informations.

L'IE a besoin de personnel pour mener à bien sa pratique. Donner un véritable statut aux collaborateurs qui occupent les postes de surveillance, est essentiel. L'IE est de plus souvent perçue comme "l'antichambre » de la Direction Générale, laissant planer le doute d'une sorte "d'espionnage interne " de la Direction sur les salariés. Le nombre de salariés dédiés à la fonction d'IE et l'implication du dirigeant est fonction de la taille de l'entreprise (Lackman et al., 2000). Ce constat est à relativiser toutefois, car dans le cas d'une PME, par exemple, l'IE est pratiquée par des collaborateurs qui ne sont pas nécessairement identifiés et sans réel outil mis à leur disposition. Lenz et Engledow (1986) indiquent que les services de 
veilles ou d'IE comptent de un à sept employés. Prescott (1999) comptabilise trois personnes à temps plein, une à temps partiel et une secrétaire dans l'équipe de surveillance de l'entreprise étudiée. Ces deux auteurs indiquent également qu'un collaborateur ne peut s'improviser veilleur ou responsable d'IE.

Les spécialistes et les non spécialistes de l'information se côtoient dans l'entreprise. Le responsable d'IE est cependant le seul investigateur dans la pratique de surveillance. Un spécialiste de l'IE doit cumuler trois types de compétences (Prescott, 1999) : celles de succès (maîtrise des techniques d'acquisition, validation des sources informationnelles, l'analyse, etc.), celles qui sont complémentaires (connaissance du secteur, maîtrise des outils informatiques, etc.), et celles qualifiées d'aptitudes (curiosité, ouverture d'esprit, les capacités relationnelles, etc.). Pour Fleisher (2004), les praticiens de l'IE doivent cumuler trois types de compétences. Ils doivent avoir des aptitudes telles que la créativité, la ténacité, etc. Ils doivent avoir des capacités intellectuelles afin d'analyser les informations, de comprendre les portées stratégiques de celles-ci, etc. Ils doivent pouvoir se servir de leurs expériences professionnelles multiples afin de diversifier leurs compétences et donc d'accroître leur capacité à comprendre. Lackman et al. (2000) mettent en évidence cinq caractéristiques d'un analyste de l'IE : la créativité, l'imagination, la curiosité, la ténacité et une certaine ouverture.

Ces auteurs montrent également que la majorité des collaborateurs qui participe à la démarche d'IE, n'est pas experte de l'information et ne participe pas à la démarche à temps plein. C'est le personnel le plus aguerri et ancien, en matière d'IE, qui occupe le poste de responsable. Il est le plus à même d'avoir l'expérience, les compétences et un pouvoir de communication. Les rares experts de l'information sont employés à temps plein à l'exercice de l'IE.

Au regard de ce qui précède, les acteurs de l'IE au sein de l'entreprise sont difficiles à identifier. Tous les collaborateurs participent à l'effort d'IE, mais tous n'en ont pas la compétence et la capacité.

La revue de la littérature montre que les débats sur le personnel dédié à l'effort d'IE sont importants. Les travaux s'avèrent, à certains égards, être contradictoires. Pour certaines études, seuls les dirigeants pratiquent l'IE, alors que pour d'autres études, l'ensemble des salariés est concerné par cette fonction, quel que soit le niveau hiérarchique. Cette opposition persiste selon que l'on considère que l'IE est pratiquée par des spécialistes de l'information et qu'elle est exercée par des non-spécialistes. Ne pouvant trancher ces ambivalences par la littérature, nous émettons deux hypothèses primaires et six hypothèses secondaires (Cf. Figure 1). Nous distinguons les non-spécialistes de l'information que nous classons en fonction du niveau hiérarchique, et les spécialistes de l'information recrutés pour cette tâche, que nous classons en fonction des étapes du processus d'IE à accomplir.

Hypothèses relatives aux non-spécialistes :

- H1 : Plus une entreprise pratique une forme d'IE et plus l'IE est pratiquée par des non-spécialistes.

- H1a : Plus une entreprise pratique une forme d'IE et plus les nonspécialistes se situent au niveau du « top management » (Direction Générale). 
- H1b : Plus une entreprise pratique une forme d'IE et plus les nonspécialistes se situent au niveau du « middle management » (Cadres, etc.).

- H1c : Plus une entreprise pratique une forme d'IE plus les nonspécialistes se situent au niveau du « low management » (Salariés, etc.).

Hypothèses relatives aux spécialistes :

- H2 : Plus une entreprise pratique une forme d'IE et plus l'IE est pratiquée par des spécialistes.

- H2a : Plus une entreprise pratique une forme d'IE et plus les spécialistes sont des collecteurs d'information.

- H2b : Plus une entreprise pratique une forme d'IE et plus les spécialistes sont des analystes d'information.

- H2c : Plus une entreprise pratique une forme d'IE et plus les spécialistes sont des diffuseurs d'information.

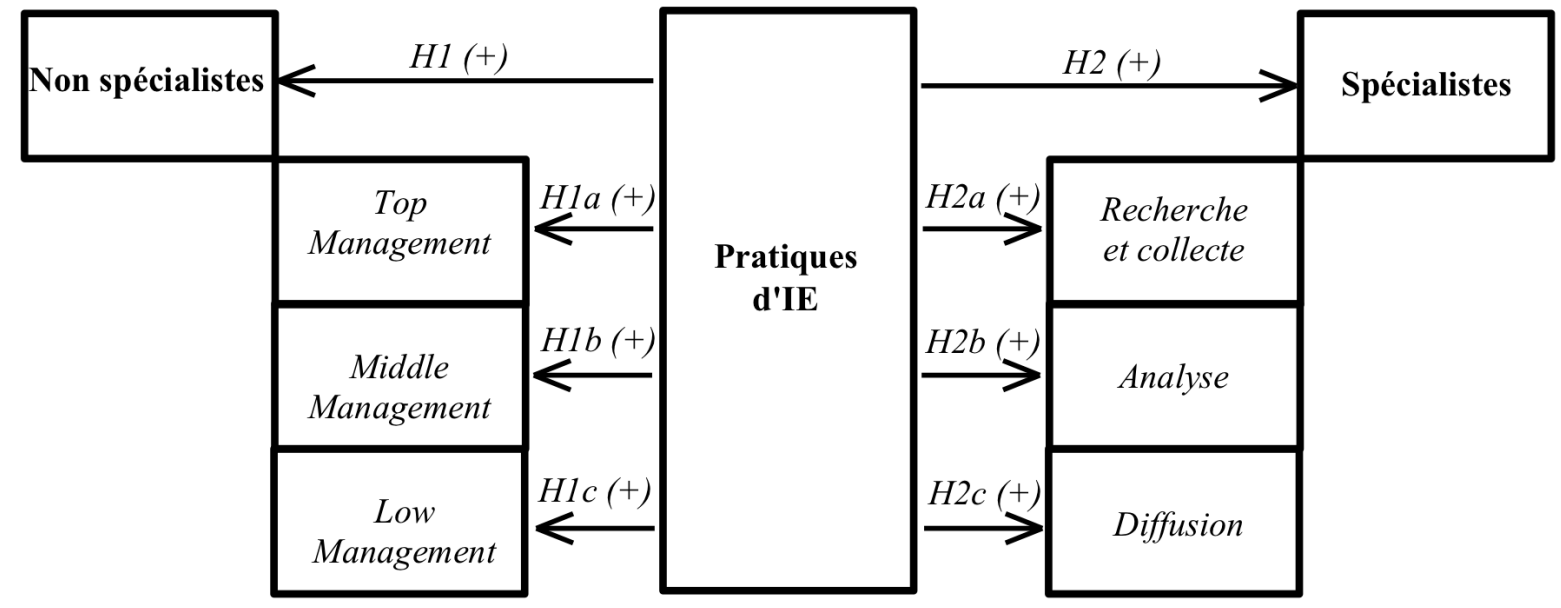

Figure 1 : Modèle théorique.

\section{Méthode}

\section{1 Échantillon}

Nous avons envoyé un questionnaire de recherche auprès de 1500 entreprises européennes (25 pays en 2007) de haute technologie, à destination des PDG, DG, gérants Directeurs d'Unité, etc. La Commission Européenne définit huit secteurs comme étant de haute technologie (Eurostat, 2005) dont trois sont relatifs, aux services (NACE 64, 72 et 73) et cinq aux produits (NACE 353, 244, 30, 32 et 33). L'échantillon a été défini de manière aléatoire, à probabilités égales, sans distinction de tailles, de chiffre d'affaires, etc. Parmi les 174 entreprises qui ont répondu (11,4\% de retours), 153 ont été retenu après épuration. 90,9 $\%$ des répondants occupent un poste à haute responsabilité. Quatre catégories d'entreprises en fonction de la taille (TPE, PE, ME et GE) montrent une homogénéité dans la répartition (environ $25 \%$ chacune). 


\subsection{Questionnaire}

Le questionnaire est constitué de huit macro-variables (communiqué sur demande). Les items sont mesurés par le biais d'échelles de Lickert de quatre modalités (de «Pas du tout d'accord » à « Tout à fait d'accord »).

\subsubsection{Variable dépendante}

La macro-variable 1 (MV1) nommée "Acteurs » s'intéresse aux acteurs de l'IE en distinguant les «Spécialistes » (AcSp) de l'information tels que les responsables de système d'information, de veille, d'IE, etc. et les « Non Spécialistes » (AcNSp).

\subsubsection{Variables indépendantes}

Les macro-variables de MV2 à MV5 ont également été utilisées pour cartographier les groupes d'entreprises selon leur niveau de pratiques d'IE.

La macro-variable 2 (MV2) nommée «Surveillances environnementales » indique les pratiquants de veilles et de renseignements envers trois groupes d'acteurs : les concurrents représentés par la variable «Veille concurrentielle » (SuCon), les clients et les fournisseurs représentés par la variable «Veille commerciale » (SuCom), et les organismes institutionnels privés et publics représentés par la variable «Veille institutionnelle » (SuIn).

La macro-variable 3 (MV4) nommée « Connaissances environnementales » indique la connaissance perçue des forces et faiblesses de deux groupes d'acteurs : les concurrents représentés par la variable "Connaissances horizontales » (CoHo), et les clients et les fournisseurs représentés par la variable « Connaissances verticales » $(\mathrm{CoVe})$.

La macro-variable 4 (MV4) nommée « Relations environnementales » indique les échanges informationnels et collaboratifs avec trois groupes d'acteurs : les concurrents représentés par la variable "Relations horizontales » (ReHo), les clients et les fournisseurs représentés par la variable "Relations verticales » (ReVe) et les organismes institutionnels privés représentés par la variable « Relations institutionnelles » (ReIn).

La macro-variable 5 (MV5) nommée « Influences environnementales » indique l'influence qu'une entreprise pense avoir sur son environnement. Trois variables composent MV4 : «Influence» (InInf) indiquant la capacité d'influence perçue (lobbying, etc.), "Promotion réseau» (InPro) correspondant à la promotion d'un réseau d'entreprises (appartenance, instigateur d'un réseau d'entreprise, etc.) et " Adhésion institutionnelle » (InAd) correspondant à l'appartenance à des clubs, des syndicats professionnels, etc. Les modalités des items ont été binéarisées pour les besoins de l'étude.

La macro-variable 6 (MV6) nommée «Structuration » indique le degré de structuration de la gestion de l'information selon deux distinctions : une "Structuration forte » (StFo) représentant une gestion informationnelle plutôt centralisée, formalisée et internalisée et une «Structuration faible » $(\mathrm{StFa})$ représentant une gestion informationnelle plutôt décentralisée, non formalisée et externalisée de l'information. Les modalités des items ont été binéarisées pour les besoins de l'étude.

La macro-variable 7 (MV7) nommée «Satisfaction informationnelle » (AdSa) mesure les niveaux de satisfaction de la gestion informationnelle. 
La macro-variable 8 (MV8) nommée «Connaissances » indique les données liées à la connaissance et au savoir dans les entreprises. Deux variables composent MV9 : "Gestion des connaissances " (ConGe) indiquant la construction, la pérennisation et la gestion des savoirs et «Activités de R\&D » (ConAc) indiquant les actions de R\&D. Les modalités des items ont été binéarisées pour les besoins de l'étude.

\subsubsection{Variables de contrôle}

La variable «Taille des entreprises » est étudiée en fonction du chiffre d'affaires et de l'effectif conformément à la législation européenne $(2003 / 361 / \mathrm{CE})$ : Micro et TPE $(\mathrm{CA}>2000 \mathrm{~K} €$, Effectif $<10$ ), PE (CA entre 2000 et $10000 \mathrm{~K} €$, effectif entre 10 et 50), ME (CA entre 10000K€ et 50000, effectif entre 50 et 250), GE (CA >50000, effectif >250).

La variable «Indépendance patrimoniale » aborde tour à tour l'appartenance à un groupe, le nombre d'actionnaires dans les entreprises et le nombre de filiales de l'entreprise.

La variable «Leadership » indique les positions concurrentielles occupées par les entreprises ayant répondu.

Tous les tests de validités convergentes et discriminantes effectués sur ces échelles de mesures sont corrects.

\subsection{Traitements des données}

L'analyse en composante principale (ACP) a permis de construire les variables de l'étude qui ont été validées par l'analyse factorielle confirmatoire (AFC). Les rotations orthogonales et obliques ont permis de retenir les scores factoriels restituant le plus d'informations. Les analyses typologiques et discriminantes ont construit et validé quatre groupes d'entreprises selon leur niveau de pratiques $d^{\prime} I E$, puis trois fonctions discriminantes. L'étude des scores et barycentres a permis de révéler les pratiques plus ou moins fortes des variables considérées par groupe. L'analyse de la variance (ANOVA) a mesuré le degré de significativité d'une variable explicative sur une variable à expliquer. Les tests de comparaisons multiples ou TCM (tests $t$, Tukey où $n$ inégaux, Scheffe, LSD et Bonferroni) ont distingué l'implication des groupes pour les variables étudiées. Enfin, l'analyse de régression multiple pas-à-pas ascendant, par groupe, a déterminé les variables explicatives de la présence d'acteurs spécialistes de l'information (AcSp) et non spécialistes (AcNSp).

\section{Résultats}

\subsection{Quatre groupes constitués}

Quels sont les degrés de pratiques de renseignement des entreprises?

Les analyses typologiques et discriminantes montrent que l'échantillon peut être significativement scindé en quatre groupes $(\mathrm{p}<0,001)$. Nous inscrivons entre parenthèses le signe des barycentres (noté G).

Le groupe 1 (NP) regroupe majoritairement 40 moyennes entreprises qui ne pratiquent aucune forme de surveillance. Les activités de veilles institutionnelles, commerciales et concurrentielles $(G<0)$ sont quasi-inexistantes. Ces entreprises méconnaissent leur environnement concurrentiel et commercial $(G<0)$ et ont peu de relations partenariales et 
d'alliance $(G<0)$. Elles sont peu influentes, se positionnent peu sur des réseaux d'entreprises ou institutionnels, et adhèrent peu à des clubs et à des syndicats professionnels $(G<0)$.

Le groupe 2 (VNC) regroupe majoritairement 39 petites entreprises qui pratiquent une veille dite «non coopérative ». Elles pratiquent des activités de veilles concurrentielles et commerciales $(G>0)$, mais peu de veille institutionnelle $(G<0)$. Elles méconnaissent leur environnement concurrentiel et commercial $(\mathrm{G}<0)$, et ont peu de relations partenariales et $\mathrm{d}$ 'alliance $(\mathrm{G}<0)$. Elles sont peu influentes, se positionnent peu sur des réseaux d'entreprises ou institutionnels et adhèrent peu à des clubs et à des syndicats professionnels $(G<0)$.

Le groupe $3(\mathrm{VC})$ regroupe majoritairement 37 petites entreprises qui pratiquent une veille «d'alliance ». Elles pratiquent des activités de veilles concurrentielles et commerciales $(G>0)$, mais peu de veille institutionnelle $(G<0)$. Elles connaissent leur environnement concurrentiel et commercial $(G>0)$, et ont des relations partenariales et d'alliance $(G>0)$. Elles sont peu influentes $(G<0)$, mais se positionnent dans des réseaux d'entreprises ou institutionnels, et adhèrent à des clubs et à des syndicats professionnels $(G>0)$.

Le groupe 4 (IEP) regroupe majoritairement 37 grandes entreprises qui pratiquent une IE dite « partenariale ». Les activités de veilles institutionnelles, commerciales et concurrentielles $(G>0)$ sont fortes. Ces entreprises connaissent leur environnement concurrentiel et commercial $(G>0)$ et ont de multiples relations partenariales $(G>0)$, mais pas d'alliance $(G<0)$. Elles sont influentes, se positionnent dans des réseaux d'entreprises ou institutionnels, et adhèrent à des clubs et à des syndicats professionnels $(G>0)$.

Quelle variable permet de distinguer une pratique de veille d'une démarche d'IE ?

L'analyse discriminante montre l'importance des variables liées à la capacité d'influence de l'environnement. C'est cette capacité d'influence qui fait la distinction entre la veille, qui n'en dispose pas, et l'IE, dont c'est une des composantes majeures. Nous inscrivons entre parenthèses, les barycentres. Trois fonctions discriminantes ont été révélées :

- une fonction nommée "surveillance réactive », qui a un pouvoir discriminant de $27,4 \%$, et qui est essentiellement attribuée aux entreprises pratiquant une veille ouverte $(2,096)$;

- une fonction nommée "surveillance proactive », qui a un pouvoir discriminant de $31,9 \%$, et qui est attribuée aux entreprises pratiquant une veille fermée $(1,307)$ ou ouverte $(0,980)$ ou une démarche d'IE $(0,026)$;

- une fonction nommée "surveillance interactive », qui a un pouvoir discriminant de $36,8 \%$, et qui est attribuée uniquement aux entreprises pratiquant l'IE $(2,483)$.

\subsection{Gestionnaires des informations}

Qui gère les informations dans les entreprises?

\subsubsection{Les non-spécialistes de l'information}

Nous entendons et catégorisons par «non-spécialistes », les salariés qui n'ont pas suivi une formation spécifique sur la gestion des informations ou les techniques de renseignement. Il existe une différence significative (Test de Fisher où $p<0,05)$ entre les groupes en ce qui 
concerne la présence de non-spécialistes de l'information (AcNSp). Par la suite, nous inscrivons entre parenthèses, le niveau de signification des tests de Student.

\section{Concernant les Directeurs Généraux ...}

La collecte $(p<0,05)$ et la diffusion $(p>0,05)$ des informations sont fortement réalisées par les Directeurs Généraux, surtout pour les entreprises qui pratiquent une forme de veilles VNC et VC. Les groupes IEP et NP se situent au second plan. Alors, les DG sont plus des collecteurs que des diffuseurs quel que soit le groupe.

En matière de collecte, seul le test $\operatorname{LCD}(p<0,05)$ montre que le groupe VNC se distingue des groupes NP et IEP, et que le groupe VC se différencie des groupes NP et IEP. $\mathrm{Si}$ nous regroupons les «veilleurs » $(\mathrm{VNC}+\mathrm{VC})$, ces derniers se distinguent de tous les autres groupes (tous TCM où $\mathrm{p}<0,05$ ). Plus l'entreprise s'inscrit dans une activité de veille, plus la collecte des informations est réalisée par les DG.

En matière de diffusion, tous les TCM $(\mathrm{p}<0,05)$ montrent que le groupe VC se distingue du groupe IEP. Si nous regroupons les «veilleurs ", ces derniers se distinguent du groupe IEP (tous TCM où $\mathrm{p}<0,05$ ). Les activités de veilles avancées semblent être associées à une diffusion informationnelle réalisée par les DG, mais les résultats ne nous permettent pas de trancher cette observation $(p>0,05)$.

L'hypothèse H1a (Plus une entreprise pratique une forme d'IE et plus les nonspécialistes se situent au niveau du « top management ») est partiellement validée.

Concernant les cadres ...

La collecte et la diffusion $(\mathrm{p}<0,05)$ des informations sont fortement réalisées par les cadres, surtout dans le groupe IEP. Les groupes NP, VNC et VC se situent au second plan. Les cadres sont alors plus des collecteurs que des diffuseurs, et s'impliquent plus fortement dans ces deux activités que les DG. Les TCM montrent qu'aucun des groupes ne se distingue d'un autre $(p>0,05)$, aussi bien en matière de collecte que de diffusion des cadres. Nous ne pouvons pas conclure à une prédominance d'un groupe par rapport à un autre.

L'hypothèse H1b (Plus une entreprise pratique une forme d'IE et plus les nonspécialistes se situent au niveau du « middle management ») est partiellement validée.

Concernant les salariés ...

La collecte et la diffusion $(\mathrm{p}<0,05)$ des informations réalisées par les salariés sont plus faiblement pratiquées surtout par le groupe VC contrairement aux groupes VNC, NP et IEP. Les salariés sont plus des collecteurs que des diffuseurs et s'impliquent moins fortement dans ces deux activités, que les DG ou les cadres.

En matière de collecte, aucun groupe ne se distingue d'un autre (tous TCM où $p>0,05$ ).

En matière de diffusion, les TCM $(p<0,05)$ montrent que le groupe VNC se distingue du groupe VC. Le groupe VC se caractérise par une diffusion faible de la part des salariés, et se distingue du groupe VNC. Les résultats ne nous permettent pas toutefois de trancher sur une prédominance d'un groupe sur un autre en la matière $(p>0,05)$.

L'hypothèse H1c (Plus une entreprise pratique une forme d'IE, plus les nonspécialistes se situent au niveau du « low management ») est rejetée. 
L'hypothèse H1 (Plus une entreprise pratique une forme d'IE et plus l'IE est pratiquée par des non-spécialistes) est partiellement validée.

\subsubsection{Les spécialistes de l'information}

Nous entendons et catégorisons par "spécialistes ", les salariés qui ont suivi une formation spécifique sur la gestion des informations ou les techniques de renseignement. Il existe une différence significative (Test de Fisher où $p<0,001$ ) entre les groupes en ce qui concerne la présence de spécialistes de l'information (AcSp). Cette variable est fortement présente dans le groupe IEP contrairement à tous les autres groupes. Cette distinction subsiste significativement lorsque l'on regroupe les entreprises veilleuses (VNC + VC) : plus les pratiques des entreprises se rapprochent de l'IE et plus la présence de spécialistes de l'information est forte.

Nous inscrivons, par la suite, entre parenthèses, le niveau de signification des tests de Student.

La collecte et la diffusion $(p<0,001)$ des informations réalisées par les spécialistes de l'information sont beaucoup plus fortement pratiquées pour le groupe IEP que dans les groupes VNC, VC et NP. La présence de spécialistes de l'information est une caractéristique distinctive du groupe IEP par rapport aux autres groupes. Les spécialistes sont plus des collecteurs que des diffuseurs, et s'impliquent d'une manière équivalente aux cadres.

En matière de collecte, tous les TCM $(\mathrm{p}<0,01)$ montrent que le groupe IEP se distingue des autres groupes. Si nous regroupons les «veilleurs", ces derniers se distinguent du groupe IEP (tous TCM où $\mathrm{p}<0,001$ ). Par conséquent, plus l'entreprise s'approche vers une pratique d'IE, plus les informations sont collectées par des spécialistes de l'information.

En matière de diffusion, les TCM montrent que le groupe IEP se distingue des groupes $\mathrm{NP}$ et $\mathrm{VC}(\mathrm{p}<0,001)$ ainsi que VNC $(\mathrm{p}<0,05)$. Si nous regroupons les « veilleurs », ces derniers se distinguent $d u$ groupe IEP (tous TCM où $p<0,001$ ). Plus l'entreprise s'approche d'une pratique d'intelligence économique, plus les informations sont diffusées par des spécialistes de l'information.

Nous pouvons noter que les résultats pour l'étape « analyse des informations » ne se révèlent pas significatives. Nous pouvons donc en conclure que l'analyse des informations n'est pas réalisée par les spécialistes.

L'hypothèse H2 (Plus une entreprise pratique une forme d'IE et plus l'IE est pratiquée par des spécialistes) est validée.

L'hypothèse H2a (Plus une entreprise pratique une forme d'IE et plus les spécialistes sont des collecteurs d'information) est validée.

L'hypothèse $\mathrm{H} 2 \mathrm{~b}$ (Plus une entreprise pratique une forme d'IE et plus les spécialistes sont des analystes d'information) est rejetée.

L'hypothèse H2c (Plus une entreprise pratique une forme d'IE et plus les spécialistes sont des diffuseurs d'information) est validée.

\subsection{Les déterminants de la présence (ou non) de spécialistes de l'information}

Quelles sont les variables qui influencent la présence (ou non) de spécialistes de l'information dans une entreprise? 
Les acteurs de l'intelligence économique dans les entreprises : le cas des entreprises européennes de hautes technologies

Deux modèles de régression ont été réalisés (Cf. Tableau 1):

- l'un met en relation les variables explicatives et la variable à expliquer « absence de spécialistes de l'information » (AcNSp),

- le second met en relation les variables explicatives et la variable à expliquer « présence de spécialistes de l'informatio » (AcSp).

\begin{tabular}{|c|c|c|c|c|c|c|c|c|c|}
\hline & \multicolumn{4}{|c|}{$\begin{array}{l}\text { Modèle «Absence de } \\
\text { spécialistes " }\end{array}$} & \multicolumn{4}{|c|}{$\begin{array}{l}\text { Modèle «Présence de } \\
\text { spécialistes " }\end{array}$} \\
\hline & & NP & VNC & VC & IEP & NP & VNC & $\mathrm{VC}$ & IEP \\
\hline $\begin{array}{c}\text { Macro- } \\
\text { variables }\end{array}$ & Variables & & & & & & & & \\
\hline \multirow{3}{*}{$\begin{array}{l}\text { Surveillances } \\
\text { environnement. }\end{array}$} & $\begin{array}{l}\text { Veille } \\
\text { concurrentielle }\end{array}$ & & & $\begin{array}{l}0,347 \\
(* *)\end{array}$ & & & & $\begin{array}{l}0,482 \\
\left({ }^{*}\right)\end{array}$ & $\begin{array}{l}0,439 \\
(* * *)\end{array}$ \\
\hline & $\begin{array}{l}\text { Veille } \\
\text { commerciale }\end{array}$ & & & $\begin{array}{l}0,811 \\
(*)\end{array}$ & & & & $\begin{array}{l}0,273 \\
(\star * *)\end{array}$ & \\
\hline & $\begin{array}{l}\text { Veille } \\
\text { institutionnelle }\end{array}$ & & $\begin{array}{l}-0,362 \\
(* * *)\end{array}$ & & & & & & \\
\hline \multirow{2}{*}{$\begin{array}{l}\text { Connaissances } \\
\text { environnement. }\end{array}$} & $\begin{array}{l}\text { Connaissances } \\
\text { horizontales }\end{array}$ & $\begin{array}{l}0,295 \\
(* *)\end{array}$ & $\begin{array}{l}0,543 \\
(* *)\end{array}$ & & & & & & \\
\hline & $\begin{array}{l}\text { Connaissances } \\
\text { verticales }\end{array}$ & & $\begin{array}{l}-0,404 \\
(* *)\end{array}$ & & & & & $\begin{array}{l}0,238 \\
(* *)\end{array}$ & \\
\hline \multirow{3}{*}{$\begin{array}{l}\text { Relations } \\
\text { environnement. }\end{array}$} & $\begin{array}{l}\text { Relations } \\
\text { horizontales }\end{array}$ & & & $\begin{array}{l}--0,673 \\
\left({ }^{*}\right)\end{array}$ & & $\begin{array}{l}-0,381 \\
\left({ }^{*}\right)\end{array}$ & & & \\
\hline & $\begin{array}{l}\text { Relations } \\
\text { verticales }\end{array}$ & & & $\begin{array}{l}0,467 \\
(* *)\end{array}$ & & & & $\begin{array}{l}-0,231 \\
(* * *)\end{array}$ & \\
\hline & $\begin{array}{l}\text { Relations } \\
\text { institutionnelles }\end{array}$ & & $\begin{array}{l}-0,692 \\
(* *)\end{array}$ & $\begin{array}{l}0,235 \\
(* * *)\end{array}$ & & & & $\begin{array}{l}0,160 \\
(* * *)\end{array}$ & $\begin{array}{l}0,273 \\
(* * *)\end{array}$ \\
\hline \multirow{3}{*}{$\begin{array}{l}\text { Influences } \\
\text { environnement. }\end{array}$} & Influence & $\begin{array}{l}0,734 \\
(* *)\end{array}$ & & $\begin{array}{l}0,902 \\
(*)\end{array}$ & $\begin{array}{l}-0,498 \\
(* * *)\end{array}$ & & & & \\
\hline & $\begin{array}{l}\text { Promotion } \\
\text { réseau }\end{array}$ & & $\begin{array}{l}-0,364 \\
(* * *)\end{array}$ & & & & & & \\
\hline & $\begin{array}{l}\text { Adhésion } \\
\text { institutionnelle }\end{array}$ & $\begin{array}{l}0,467 \\
(* * *)\end{array}$ & & $\begin{array}{l}-0,568 \\
\left({ }^{* *}\right)\end{array}$ & & $\begin{array}{l}-0,244 \\
(* *)\end{array}$ & & & \\
\hline \multirow{2}{*}{ Structuration } & $\begin{array}{l}\text { Structuration } \\
\text { forte }\end{array}$ & $\begin{array}{l}-0,989 \\
(* *)\end{array}$ & & & & $\begin{array}{l}0,995 \\
(*)\end{array}$ & & $\begin{array}{l}0,454 \\
\left({ }^{*}\right)\end{array}$ & $\begin{array}{l}0,554 \\
\left({ }^{*}\right)\end{array}$ \\
\hline & $\begin{array}{l}\text { Structuration } \\
\text { faible }\end{array}$ & $\begin{array}{l}0,661( \\
\left.{ }^{*}\right)\end{array}$ & & & & $\begin{array}{l}-0,412 \\
(*) \\
\end{array}$ & & & $\begin{array}{l}-0,460 \\
(*) \\
\end{array}$ \\
\hline \multicolumn{2}{|c|}{ Satisfaction informationnelle } & & & $\begin{array}{l}-0,701 \\
(*)\end{array}$ & & & & & \\
\hline \multirow{4}{*}{ Connaissances } & $\begin{array}{l}\text { Gestion des } \\
\text { connaissances }\end{array}$ & & $\begin{array}{l}-0,548 \\
(*)\end{array}$ & $\begin{array}{l}0,225 \\
(* * *)\end{array}$ & & $\begin{array}{l}0,177 \\
(* * *)\end{array}$ & & & $\begin{array}{l}0,494 \\
(* * *)\end{array}$ \\
\hline & $\begin{array}{l}\text { Activités de } \\
\text { R\&D }\end{array}$ & & & & & & & & \\
\hline & Tests de Fisher & $\begin{array}{l}6,105 \\
(*)\end{array}$ & $\begin{array}{ll}3,023 \\
(*)\end{array}$ & $\begin{array}{l}10,712 \\
\left(^{*}\right)\end{array}$ & $\begin{array}{l}7,849 \\
(*)\end{array}$ & $\begin{array}{l}17,956 \\
(*)\end{array}$ & $\begin{array}{l}2,223 \\
(\mathrm{~ns})\end{array}$ & $\begin{array}{l}22,139 \\
(*)\end{array}$ & $\begin{array}{l}8,549 \\
(*)\end{array}$ \\
\hline & $\mathrm{R}^{2}$ & 0,774 & 0,611 & 0,858 & 0,655 & 0,797 & 0,372 & 0,881 & 0,79 \\
\hline
\end{tabular}

Tableau 1 : Analyse de régression multiple pas à pas . Lecture : (ns) non significatif ; $\left({ }^{*}\right)$

$$
\left.\left.\mathrm{p}<0,001 ;{ }^{* *}\right) \mathrm{p}<0,01 ;{ }^{* * *}\right) \mathrm{p}<0,05 .
$$

Ces deux modèles ont été étudiés pour chacun des quatre groupes constitués en fonction du degré de pratique de renseignement des entreprises. 


\section{Pour le groupe NP}

Plus les firmes ont faiblement structuré leur gestion des informations, connaissent leurs concurrents, ont une capacité d'influence sur leur environnement et adhérent à des clubs et syndicats professionnels, et plus la gestion des informations est gérée par des non spécialistes.

Moins les firmes entretiennent de relations d'alliances, adhérent à des clubs et syndicats professionnels, et ont faiblement structuré leur gestion des informations, et plus la gestion des informations est gérée par des spécialistes. Plus les firmes ont faiblement structuré leur gestion des informations et gèrent leurs connaissances, et plus la gestion des informations est gérée par des spécialistes.

\section{Pour le groupe VNC}

Moins les firmes pratiquent une veille institutionnelle, connaissent leurs clients et fournisseurs, entretiennent de relations institutionnelles, participent à des réseaux d'entreprises et gèrent leurs connaissances, et plus la gestion des informations est gérée par des non-spécialistes. Plus les firmes connaissent leurs concurrents, et plus la gestion des informations est gérée par des non-spécialistes.

Pour le groupe VC

Plus les firmes pratiquent une veille commerciale et concurrentielle, entretiennent des relations partenariales et institutionnelles, ont une capacité d'influence sur leur environnement et gèrent leurs connaissances, et plus la gestion des informations est gérée par des non-spécialistes. Moins les firmes entretiennent des relations d'alliances, adhérent à des clubs et syndicats professionnels et sont satisfaites de leur gestion informationnelle, et plus la gestion des informations est gérée par des non-spécialistes.

Plus les firmes, pratiquent une veille commerciale et institutionnelle, connaissent leurs clients et fournisseurs, entretiennent des relations institutionnelles et ont fortement structuré leur gestion des informations, et plus la gestion des informations est gérée par des spécialistes. Moins les firmes, entretiennent des relations partenariales, et plus la gestion des informations est gérée par des spécialistes.

Pour le groupe IEP

Moins les firmes ont une capacité d'influence sur leur environnement, et plus la gestion des informations est gérée par des non-spécialistes. Plus les firmes pratiquent une veille concurrentielle, entretiennent de relations institutionnelles, ont fortement structuré leur gestion des informations et gèrent leurs connaissances, et plus la gestion des informations est gérée par des spécialistes.

\section{Discussions}

Qui pratique l'IE dans l'entreprise ? Les résultats des travaux académiques antérieurs n'apportent pas les mêmes réponses à cette question, oscillant entre adéquation et opposition. Nos résultats indiquent qu'il existe un compromis entre les différentes études et entre les diverses visions d'appréhender la pratique de l'IE. Trois apports peuvent être retenus dans notre étude. 
Notre premier apport consiste à avoir identifié quatre groupes d'entreprises afin de caractériser leur niveau de surveillance. Nous avons identifié les entreprises qui ne pratiquent aucune forme de veille (NP), conformément aux travaux antérieurs (Larivet, 2002, Levet, 2002, Salles, 2003). Nous avons détecté deux types de veilles : les entreprises qui pratiquent une forme de veille dite "non-coopérative» $(\mathrm{VNC})$; et celles qui exercent une veille dite « $\mathrm{d}^{\prime}$ alliance » $(\mathrm{VC})$. Les travaux antérieurs corroborent les résultats en montrant la faible capacité d'influence (Larivet, 2002) pour les deux types de veille. Pour les veilleurs «non-coopératifs", les relations coopératives quasiment inexistantes ont été développées par Levet (2002) et Salles (2003). Les auteurs parlent «d'entreprises tayloriennes». Pour les veilleurs "alliés », notre étude se distingue des "entreprises commerciales » développées par Salles (2003), car nos résultats montrent une focalisation sur les relations coopératives horizontales (entre rivaux). Enfin, nous avons déterminé les PME pratiquant une forme d'IE dite «partenariale» (IEP). Les études précédentes appuient leurs résultats en indiquant que l'exercice d'une surveillance multiple et d'une capacité d'influence forte distinguent une démarche d'IE d'une simple démarche de veille (Larivet, 2002). Nos résultats s'avèrent être conformes aux conclusions de Salles (2003) sur les « entreprises cognitives ».

Notre deuxième apport consiste à avoir identifié les acteurs de l'IE dans l'entreprise. Les experts de l'information ne sont pas les seuls salariés actifs dans le processus d'IE. En effet, les Directeurs Généraux s'investissent aussi dans la recherche, la collecte, l'analyse et la diffusion des informations. Leur activité de surveillance se focalise essentiellement sur les perspectives stratégiques pour l'entreprise. Ce sont les cadres toutefois qui participent le plus à l'ensemble des étapes composant la démarche d'IE. Nos résultats montrent également que les salariés restent encore peu impliqués dans l'exercice du renseignement. Comme l'indique nombre d'auteurs, le dirigeant a toujours la main sur la fonction d'IE. Cela se vérifie dans nos conclusions, car les dirigeants qui pratiquent l'IE sont plus nombreux dans les PME/PMI de notre échantillon. Ce constat reste paradoxal car, comme l'indique Gilad et Gilad (1985), un des avantages de la centralisation de l'IE est de bénéficier d'un personnel motivé, disponible et formé. Le personnel est formé par l'emploi d'expert, mais reste insuffisamment exploité par le reste des salariés. Notre étude montre que bien que l'implication du dirigeant est forte dans le processus d'IE, la sensibilisation à cette pratique

envers les salariés reste faible. Cette culture de renseignement est encore marginale dès lors que l'on considère les salariés hors cadre. La proposition de formations, voire d'incitations financières, permettrait de renforcer cette démarche collective, en encourageant et motivant l'ensemble des salariés. Force est de constater que l'IE n'est pas une démarche participative, à tous les niveaux hiérarchiques, contredisant les recommandations de Rouach (2008).

Les experts de l'information sont néanmoins les principaux acteurs du processus d'IE dans l'entreprise, mais ils restent autant impliqués dans la démarche que les cadres. Ce résultat est conforme aux travaux antérieurs. En d'autres termes, l'implication des cadres non-spécialistes et des experts dans l'ensemble des étapes du processus d'IE, reste la même. Nos résultats indiquent également que l'analyse des informations est peu réalisée par les experts. Il est intéressant de voir qu'il existe un mélange de centralisation et de décentralisation des pratiques d'IE. L'exercice de l'IE reste concentré sur le dirigeant et les experts, mais se décentralise vers les cadres. 
Rappelons que pour Lackman et al. (2000) et Bournois et Romani (2000), l'IE est centralisée au niveau de la Direction Générale. Stubbart (1982) indiquait également l'existence d'IE décentralisée au sein de chaque fonction. Les cadres sont donc perçus comme un relais du processus d'IE. Ils se trouvent à l'interface entre «top mangement » et «low management». Ils sont donc plus à même de faire circuler les informations, de les comprendre, etc., afin de mieux cibler les besoins internes. Il s'avère que leur pratique d'IE est davantage tournée vers la gestion opérationnelle de leurs activités et de leurs équipes. Par conséquent, nos résultats rejoignent ceux de Terry (1977) qui préconise que les entreprises doivent disposer à la fois d'entités centralisées et décentralisées. De plus, nos conclusions nous conduisent en partie à conforter les thèses de Lackman et al. (2000), Prescott (1999) et Fleisher (2004), qui montrent que l'expert doit réaliser l'ensemble du processus d'IE en raison de ses compétences, sa maîtrise technique, son ouverture d'esprit, etc.

Notre troisième apport concerne l'identification des variables qui influencent la présence (ou non) d'experts de l'information dans une entreprise. Nos résultats montrent qu'une firme qui structure peu son processus d'IE (plutôt décentralisé, externalisé et non formalisé) n'emploie pas d'experts. À contrario, une firme qui structure fortement son processus d'IE (plutôt centralisé, internalisé et formalisé) emploie nombre d'experts. Ces résultats confortent les études antérieures qui caractérisent le processus d'IE par une gestion des informations plutôt centralisée, internalisée et formalisée (Bournois et Romani, 2000). De plus, il apparait que la présence d'experts au sein de l'entreprise permet de construire une démarche de surveillance structurée et autonome.

L'IE joue alors son rôle majeur dans les firmes, à savoir être un pont entre $l^{\prime}$ environnement externe et interne à l'entreprise et une interface entre les acteurs de métiers et de niveaux hiérarchiques différents.

\section{Conclusion}

Cette étude montre que spécialistes et non-spécialistes de l'information se côtoient dans l'entreprise. Le rôle des cadres a souvent été sous-évalué dans les recherches antérieures. Le dirigeant détient toujours les activités stratégiques du renseignement, alors que les cadres exercent une IE plutôt opérationnelle. Enfin, les experts de l'information jouent le rôle d'interface entre les différents niveaux hiérarchiques et structurent le processus global d'IE. Notre étude souffre toutefois de certaines limites qui nécessitent des travaux futurs d'approfondissement. L'analyse de régression ne conclue pas sur une relation causale entre la présence (ou non) de spécialistes de l'information et les activités de surveillance. De plus, par sa pratique stratégique voire tabou, l'IE n'a pu être étudiée que dans son ensemble pour bien en comprendre les mécanismes. De par notre méthodologie choisie (statique), il serait important de mener une étude de cas afin de rendre compte du processus d'IE en tant que tel, sur d'autres secteurs et d'autres zones géographiques. Ces études futures pourraient notamment approfondir les politiques d'incitations et de motivation des salariés en matière d'IE, ainsi que du partage des tâches de surveillance entre l'ensemble des salariés. De même, il semble pertinent de procéder à une segmentation fine des populations et de distinguer les sous-populations stratégiques et les sous-populations non-stratégiques. Ainsi, il serait pertinent de s'interroger sur les pratiques de gestion de ressources humaines, en fonction de leur nature et de leurs publics (notion de Bundle) 


\section{Bibliographie}

Achard, P., (2005), La dimension humaine de l'intelligence économique, Hermès science publications, Paris.

Baumard, P., (2000), "Analyse stratégique, mouvements, signaux concurrentiels et interdépendance", Dunod, Paris,

Beaupre, D. et J. Cloutier, (2007), "La Gestion à Haute Performance dans la Fonction Publique Québécoise: Pratiques Mobilisatrices et Cohérence", Relations Industrielles, n62 (3), pp.516539.

Becker, B. E. et M. A. Huselid, (2006), "Strategic Human Resources Management: Where Do We Go from Here?", Journal of Management, n³2 (6), pp.898-925.

Bournois, F. et P. J. Romani, (2000), L'intelligence stratégique dans les grandes entreprises françaises, Economica, Paris.

Chung, W., H. Chen, F. Jay et J. Nunamaker, (2002), "Business Intelligence Explorer: A Knowledge Map Framework for Discovering Business Intelligence on the Web", Eller College of Business and Public Administration The University of Arizona.,

Collis, D. J. et C. A. Montgomery, (1995), "Competing on Resources", Harvard Business Review, n73 (4), pp.118-128.

Desreumaux, A., (1993), Stratégie, Dalloz, Paris.

Diffenbach, J., (1983), "Corporate Environmental Analysis in Large US Corporations", Long Range Planning, n`16 (3), pp.107-116.

Dou, H., (1995), Veille Technologique et Compétitivité: L'intelligence économique au service du développement industriel. , Dunod, Paris,

Eisenhardt, K. M. et J. A. Martin, (2000), "Dynamic Capabilities: What are they?", Strategic Management Journal, n²1 pp.1105-1121.

Fahey, L. et W. R. King, (1977), "Environmental Scanning for Corporate Planning", Business Horizons, n²0 (4), pp.61-71.

Fleisher, C. S., (2004), "Competitive Intelligence Education: Competencies, Sources, and Trends", Information Management Journal, n³8 (2), pp.56-63.

Gilad, B. et T. Gilad, (1985), "A Systems Approach to Business Intelligence", Business Horizons, n²8 (5), pp.65-70.

Hamel, G. et C. Prahalad, (1995), La conquête du futur, Interéditions, Paris.

Huselid, M., (2011), "The Impact of Human Resource Management Practices on Turnover, Productivity, and Corporate Financial Performance", Academy of Management Journal, $\mathrm{n}^{\circ} 38$ (3), pp.635-672.

Jain, S. C., (1984), "Environmental Scanning in US Corporations", Long Range Planning, nº17 (2), pp.117-128.

Klein, H. E. et R. E. Linneman, (1984), "Environment Assessment: an International Study of Corporate Practice", The Journal of Business Strategy, $n^{\circ} 5$ (1), pp.66-75.

Lackman, C. L., K. Saban et J. M. Lanasa, (2000), "Organizing the Competitive Intelligence Function: A Benchmarking Study", Competitive Intelligence Review, n¹1 (1), pp.17-27.

Larivet, S., (2002), Les réalités de l'intelligence économique en PME, QEOPS, Thèse de Doctorat en sciences de gestion, Université de Toulon et du var,

Larivet, S., (2002), "Les réalités de l'intelligence économique en PME", QEOPS, Université de Toulon et du var, (Thèse de doctorat en sciences de gestion), 
Lenz, R. T. et J. L. Engledow, (1986), "Environmental Analysis Units and Strategic Decision Making: a Field Study of Selected Leading-Edge Corporations", Strategic Management Journal, n`7 (1), pp.69-89.

Levet, J. L., (2002), Les pratiques de l'intelligence économique, huit cas d'entreprises, Paris.

Lönnqvist, A. et V. Pirttimäki, (2006), "The Measurement of Business Intelligence", Information Systems Management, $\mathrm{n}^{\circ} 23$ (1), pp.32-40.

Martre, H., (1994), Rapport : Intelligence économique et stratégies des entreprises, La Documentation Française, Paris.

Pearce, P. L., (1982), The social psychology of tourist behaviour, Pergamon Press,

Penrose, E. T., (1956), "Foreign Investment and the Growth of the Firm", The Economic Journal, nº6 (262), pp.220-235.

Peteraf, M. A., (2006), "The Cornerstones of Competitive Advantage: A Resource-Based View", Strategic Management Journal, $\mathrm{n}^{\circ} 14$ (3), pp.179-191.

Peters, T. et P. Waterman, (1984), Le prix de l'excellence: Le secret des meilleures entreprises, Intereditions, Paris.

Phanuel, D. et D. Levy, (2002), "Intelligence économique ou économie d'intelligence dans les PMEPMI ? L'exemple d'un département français", Actes de la XIème Conférence Internationale de Management Stratégique,

Porter, M. E., (1985), Competitive Advantage, Free Press New York.

Preble, J. F., P. A. Rau et A. Reichel, (1988), "The Environmental Scanning Practices of US Multinationals in the Late 1980's", Management International Review, n²8 pp.4-14.

Prescott, J. E., (1995), "The Evolution of Competitive Intelligence", International Review of Strategic Management, n6 pp.71-90.

Prescott, J. E., (1999), "The Evolution of Competitive Intelligence Designing a Process for Action", Competitive Intelligence Review, n`Spring pp.37-52.

Reed, R. et R. J. DeFillippi, (1990), "Causal Ambiguity, Barriers to Imitation, and Sustainable Competitive Advantage", Academy of Management Review, pp.88-102.

Rouach, D., (2008), La veille technologique et l'intelligence économique, Presses Universitaires de France, Paris.

Salles, M., (2003), Stratégie des PME et intelligence économique, Economica, Paris.

Stubbart, C., (1982), "Are Environmental Scanning Units Effective?", Long Range Planning, n¹5 (3), pp.139-145.

Teece, D. J., G. Pisano et A. Shuen, (2004), "Dynamic Capabilities and Strategic Management", Managing Strategic Innovation and Change, $\mathrm{n}^{\circ} 2$ pp.308-332.

Terry, P., (1977), "Mechanisms for Environmental Scanning", Long Range Planning, n¹0 (3), pp.2-9. 